\title{
RISCOS OCUPACIONAIS DOS AGENTES COMUNITÁRIOS DE SAÚDE DE UMA UNIDADE BÁSICA DE SAÚDE DO CEARÁ
}

OCCUPATIONAL RISKS OF COMMUNITY HEALTH AGENTS IN A BASIC HEALTH UNIT IN CEARÁ RIESGOS OCUPACIONALES DE LOS AGENTES COMUNITARIOS DE SALUD DE UNA UNIDAD BASICA DE SALUD DEL CEARÁ

Gisele Maria Melo Soares Arruda ${ }^{1}$

Barbara Luma Araújo Nógimo 2

Danielly Maia de Queiroz ${ }^{3}$

Pedro Alves de Araújo Filho 4

Como Citar: Arruda GMMS, Nógimo BLA, Queiroz DM, Araújo Filho PA.

Riscos ocupacionais dos agentes comunitários de saúde de uma

Unidade Básica de Saúde do Ceará. Sanare (Sobral, Online). 2021;20(2):08-16.

Descritores: Agentes comunitários de saúde; Saúde do Trabalhador; Estratégia Saúde da Família.

Descriptors:

Community Health Agents; Worker's health; Family Health Strategy.

Descriptores: Agentes Comunitarios de Salud; Salud del Trabajador; Estrategia Salud de La Familia

Submetido: $18 / 09 / 2019$

Aprovado: $10 / 08 / 2021$

Autor(a) para Correspondência: Gisele Maria Melo Soares Arruda Centro Universitário Unichristus Endereço: Rua Nunes Valente, 1988 apto 1102 bloco A, Aldeota, Fortaleza, Ceará CEP: 60125-035 E-mail:giselemelosoares@gmail.com

\section{RESUMO}

Este estudo teve por objetivo analisar riscos ocupacionais e medidas preventivas adotados pelos agentes comunitários de saúde (ACS) de uma Unidade Básica de Saúde (UBS) do Ceará. Trata-se de um estudo transversal, de natureza descritiva e abordagem qualitativa realizado em março de 2019, com seis ACS de uma UBS do Ceará. A pesquisa foi realizada por meio da aplicação de um questionário contendo 15 questões e por meio de entrevistas semiestruturadas e individuais. Os dados foram organizados e computados utilizando o programa Microsoft Excel 2016. Há predominância do sexo feminino na profissão e uma média de idade de 34 anos. A maioria dos ACS buscou uma formação de maior nível e está atuando no mesmo território há mais de cinco anos, sendo um grupo de baixa rotatividade dentro da UBS. Dentre os profissionais, $100 \%$ identificaram riscos durante a realização do seu trabalho. Evidenciou-se que o processo de trabalho desses ACS vem acarretando consequências em sua saúde e qualidade de vida. Vale ressaltar que eles afirmam conhecer e adotar medidas de segurança durante a execução do trabalho, principalmente os cuidados no trânsito e uso de proteção solar.

\footnotetext{
1. Fisioterapeuta. Mestra em Saúde Pública. Doutora em Saúde Coletiva. Universidade Federal do Ceará. E-mail: giselemelosoares@gmail.com 0RCID: https://orcid.org/0000-0002-8815-7955

2. Fisioterapeuta. Especialista em Saúde da Família e Comunidade na modalidade Residência Multiprofissional. Escola de Saúde Pública do Ceará. E-mail: barbaraluma.12@outlook.com 0RCID: http://orcid.org/0000-0003. $\underline{1284-6639}$

3. Enfermeira. Doutora em Saúde Coletiva. Universidade Estadual do Ceará. Hospital Municipal João Elísio de Holanda - Maracanaú, Ceará. E-mail: daniellymaia@yahoo.com.br ORCID: http://orcid.org/0000-0003-4780-1852 4. Cirurgião-dentista. Mestre em Saúde Coletiva. Universidade Estadual do Ceará. Secretaria Municipal de Saúde de Fortaleza. E-mail: araujofh@gmail.com 0RCID: http://orcid.org/0000-0002-4601-9220
}

Cert. de Redação Científica: Central das Revisões. Edição de texto: Karina Maria M. Machado. Revisão de provas: Texto definitivo validado pelos(as) autores(as). 


\section{ABSTRACT}

This study aimed to analyze occupational risks and preventive measures adopted by Community Health Agents (CHA) of a Basic Health Unit (BHU) in Ceará. This is a cross-sectional study of descriptive nature and qualitative approach carried out in March 2019, with six CHA from a BHU in Ceará. The research was carried out using a questionnaire with 15 questions, and semi-structured and individual interviews. The data were organized and computed using the program Microsoft Excel 2016. The female gender predominates in the profession and the average age is 34 years. Most CHAs sought higher level training and have been working in the same territory for over five years, being a group with low rotation within the BHU. $100 \%$ of the CHAs identified risks while performing their work. It became evident that the work process of these CHAs has brought consequences to their health and quality of life. It is noteworthy that they claim to know and adopt safety measures at work, especially the care in traffic and use of sunscreen.

\section{RESUMEN}

Este estudio tuvo por objetivo analizar riesgos ocupacionales y medidas de prevención adoptadas por los Agentes Comunitarios de Salud (ACS) de una Unidad Básica de Salud (UBS) del Ceará. Se trata de un estudio transversal de naturaleza descriptiva y abordaje cualitativo realizado en marzo de 2019, con seis ACS de una UBS del Ceará. La investigación fue realizada por medio de la aplicación de un cuestionario con 15 cuestiones y a través de las entrevistas semiestructuradas e individuales. Los datos fueron organizados y computados utilizando el programa Microsoft Excel 2016. Hay predominancia del sexo femenino en la profesión y una media de edad de 34 años. La mayoría de los ACS buscó una formación de mayor nivel y está actuando en el mismo territorio desde hace cinco años, siendo un grupo de baja rotación dentro de la UBS. $100 \%$ de los ACS identificaron riesgos durante la realización de su trabajo. Se evidenció que el proceso de trabajo de estos ACS viene trayendo consecuencias en su salud y calidad de vida. Se resalta que los mismos afirman conocer y adoptar medidas de seguridad durante la ejecución del trabajo, principalmente los cuidados en el tráfico y uso de protección solar.

\section{INTRODUÇÃO}

0 processo de formação e consolidação do Sistema Único de Saúde (SUS) no Brasil foi marcado pelo reconhecimento do direito universal à saúde garantido na Constituição Federal de 1988. Juntamente a isso, também se destacou a reorganização do modelo de atenção à saúde existente, em que a Atenção Primária à Saúde (APS) foi tida como eixo e modelo prioritário ${ }^{1}$.

Com a criação do Programa Saúde da Família (PSF), que posteriormente passou a ser chamado Estratégia Saúde da Família (ESF), a família tornou-se o centro dos cuidados e da organização dos processos de trabalho, exigindo, assim, que a equipe conheça o território e como cada família se reconhece e se cuida, para, dessa forma, oferecer uma melhor assistência².

Um dos membros integrantes dessa equipe é o agente comunitário de saúde $(A C S)^{2}$. 0 Programa de Agentes Comunitários de Saúde (PACS), precursor do PSF/ESF, é considerado a primeira estratégia em âmbito nacional que teve o objetivo de ampliar e fortalecer as ações de promoção e prevenção à saúde, desenvolvidas com a comunidade ${ }^{3}$.
0 ACS foi criado no Brasil na década de 90, na busca de estratégias para melhorar as condições de bem-estar e qualidade de vida da população. Essa classe de trabalhadores surgiu com a implementação de ações simples para interceder na saúde da população ${ }^{4}$, controlando os principais problemas de saúde pública de cada momento histórico.

As funções do ACS foram pensadas justamente para que esse profissional se tornasse um elo entre a Unidade Básica de Saúde (UBS) e a população dos territórios de atuação. A forma como esse trabalho é desenvolvido expõe diariamente esses profissionais a situações desafiadoras, que exigem destes habilidades tanto físicas quanto psíquicas ${ }^{5}$.

Há algum tempo vem se pensando em formas de prevenir e, consequentemente, diminuir o desgaste causado à saúde do ACS por conta de seu trabalho. Partindo do ponto de vista da saúde do trabalhador, as cargas do processo de trabalho interferem diretamente sobre a saúde do indivíduo, podendo caracterizar adoecimento orgânico e/ou psicológico ${ }^{6}$.

A proposta de estudar esta temática surge a partir da vivência profissional na residência em Saúde da Família e Comunidade. 0 fato de atuar e exercer atividades cotidianas de forma mais próxima 
a esse grupo fez surgir o interesse de conhecer um pouco mais sobre os riscos ocupacionais aos quais estão expostos e quais são as medidas preventivas adotadas. Além disso, a realização deste trabalho concretiza-se como uma forma de dar-lhes maior importância dentro da equipe e estreitar mais ainda os laços com essa classe trabalhadora.

Ao mesmo tempo em que faz parte da equipe de saúde, o ACS faz parte também da comunidade, o que o torna um dos principais elos entre os profissionais e a comunidade, reforçando sua importância dentro da ESF. Discutir sobre as atividades laborais e de saúde do profissional ACS pode ainda contribuir com a qualificação da prestação de serviços na APS, tendo em vista que, a partir desse conhecimento, poderemos construir estratégias que possam corroborar a qualificação dessas atividades.

As ações de atenção à saúde do trabalhador ainda se encontram um tanto aquém do esperado dentro das UBS. Poucos são os trabalhos voltados para a atenção e cuidados a esse público, e a abordagem dessa temática poderá contribuir para a efetivação da área da Saúde do Trabalhador no SUS, no contexto da APS, além de contribuir com a literatura sobre o tema.

Dessa forma, o presente estudo objetivou analisar os riscos ocupacionais aos quais os ACS de uma UBS estão expostos e identificar como classificam as medidas preventivas adotadas por eles.

\section{METODOLOGIA}

0 presente estudo é do tipo transversal, descritivo e de abordagem qualitativa. Foi realizado no município de Quixeramobim, localizado no Sertão Central do Ceará, que está situado na Mesorregião dos Sertões Cearenses, a 200 quilômetros da capital Fortaleza, possuindo uma população estimada em aproximadamente 80.000 habitantes e sendo a segunda maior cidade do Sertão Central?

A coleta de dados foi realizada no mês de março de 2019, com os ACS na própria Unidade Básica em que exercem suas atividades. Inicialmente, realizouse o contato com cada um de forma individual, para a explicação sobre a pesquisa e seus objetivos; após o profissional aceitar participar da pesquisa, foi feita a aplicação de um questionário e a realização de entrevista semiestruturada.

Os critérios de inclusão para a participação no estudo foram: trabalhar como ACS da UBS; ter um período mínimo de um ano de atuação naquele território; aceitar responder e entregar o questionário respondido juntamente ao Termo de Consentimento Livre e Esclarecido (TCLE) assinado. Os critérios de exclusão foram: estar afastado do trabalho durante a coleta de informações e/ou não preencher o questionário por algum motivo.

Foram excluídos do estudo um ACS que estava de licença médica e outros quatro ACS que, por algum motivo não justificado, não compareceram à coleta de dados. Sendo assim, a amostra da pesquisa foi constituida por seis profissionais que atuam como ACS na UBS em questão.

A pesquisa foi realizada por meio da aplicação de um questionário contendo 15 questões sobre a percepção de riscos ocupacionais no ambiente de trabalho, uso de equipamentos de proteção individual (EPI), conhecimento sobre prevenção de acidentes e acidentes prévios. Foram também realizadas entrevistas semiestruturadas de forma individual para conhecer o perfil socioeconômico, os riscos ocupacionais e como se classificam as medidas preventivas adotadas pelos ACS da unidade.

0 banco de dados foi construído pela digitação das respostas dos questionários, em que os dados foram organizados utilizando o programa Microsoft Excel 2016 e consolidados, com base na estatística descritiva, através de recursos de tabelas. As entrevistas foram gravadas e transcritas pelos pesquisadores. $\mathrm{Na}$ apresentação das falas, cada participante foi identificado pela sigla ACS seguida de um número, ficando ACS1, ACS2, etc.

Este estudo foi aprovado pelo Comitê de Ética em Pesquisa da Escola de Saúde Pública do Ceará (ESP/CE), com número de parecer 3.178.215/2019 e número CAAE 05540918.5.0000.5037, seguindo as normas e diretrizes da resolução 466/12 do Conselho Nacional de Saúde ${ }^{8}$.

\section{RESULTADOS E DISCUSSÃO}

Os resultados são apresentados a seguir por meio de tabelas e analisados com base na literatura vigente sobre o tema.

A Tabela 1 detalha a caracterização geral dos sujeitos do estudo. 
Tabela 1. Distribuição dos ACS quanto ao sexo e escolaridade.

\begin{tabular}{lcc}
\hline Variável & N & (\%) \\
\hline Sexo & & \\
Masculino & 0 & 0 \\
Feminino & 6 & 100 \\
Escolaridade & & \\
Nível Superior & 2 & 33 \\
Nível Técnico & 2 & 33 \\
Nível Médio & 2 & 33 \\
Outra formação & & \\
Serviço Social & 1 & 16,5 \\
Gestão Hospitalar & 1 & 16,5 \\
Técnico de enfermagem & 3 & 50 \\
Não possui & 1 & 17 \\
\hline Fonte: Dados da pesquisa. & & \\
\hline
\end{tabular}

Fonte: Dados da pesquisa.

Todas as ACS incluídas no estudo eram do sexo feminino e com uma média de idade de 34 anos. Diversos estudos mostram que a predominância na contratação de mulheres para a função de ACS vem sendo vista desde a implantação do programa, no Ceará, em $1987^{9}$.

Discute-se que a tendência para a priorização das mulheres nessa classe teve a intenção de impactar na sua condição social através da remuneração, além de inspirar outras mulheres na comunidade. Outro importante embasamento que justifica a escolha de mulheres foi a oportunidade de conseguir promover de forma mais fácil a educação em saúde para o grupo materno-infantil, tendo em vista o alto índice de morbimortalidade devido à desidratação por diarreia e resistência à amamentação como principais problemas de saúde nos anos 1980 e 1990. A orientação advinda da relação entre pares tornavase muito mais efetiva e integrada às questões culturais e linguísticas de cada território ${ }^{10}$.

Diante disso, podemos compreender que a profissão surgiu a partir do emprego de mulheres que necessitavam de trabalho e conseguiam desempenhar um papel de liderança na comunidade. Muitas delas em baixas condições econômicas e pouca qualificação, mas que deveriam ser formadas e exercer ações de urgência, pois acreditava-se que era possível alcançar resultados com a adoção de medidas simples e acertadas, daí a necessidade de orientação que conseguisse alcançar as famílias. Apesar de já existir participação masculina, e esta trazer novos olhares e perspectivas, até os dias atuais, o ACS, assim como boa parte das profissões da saúde, continua sendo uma profissão com predominância do sexo feminino ${ }^{11}$.

A média de idade evidencia a prevalência de adultos jovens na função. Uma das exigências do Ministério da Saúde é de que se tenha idade acima de 18 anos, porém não se estabelece uma idade máxima. Silva e Dalmaso ${ }^{5}$ evidenciaram que para muitos a oportunidade de ser ACS foi uma forma de ingresso no mercado de trabalho.

No que diz respeito aos aspectos sociodemográficos dos participantes do estudo, observou-se que dois ACS têm nível superior completo, dois possuem nível técnico e outros dois, nível médio. Além da profissão de ACS, um possui graduação em Serviço Social, um em Gestão Hospitalar e três possuem curso Técnico de Enfermagem.

A função de ACS, conforme previsto na lei n. ${ }^{\circ}$ 11.350/2006, exige apenas a conclusão do ensino fundamental como pré-requisito para a atuação. No entanto, no presente estudo, os resultados apontam que a maioria dos ACS buscou uma formação de maior nível, valendo ressaltar que todas na área da saúde, o que mostra além do comprometimento com a função, uma contribuição capaz de qualificar a atuação desses profissionais em suas ações cotidianas, tendo em vista que, dessa forma, dispõem de conhecimentos mais amplos ${ }^{12}$.

Esse resultado é condizente com outros autores que explanaram sobre os níveis de escolaridade do profissional ACS, revelando-nos que a maioria dos trabalhadores da APS possuem níveis fundamental e médio completos. No entanto, Santos et al. ${ }^{13}$ nos mostram que apesar da grande importância e responsabilidade do ACS dentro da equipe, ainda é totalmente desarmônica a diferença salarial entre estes e o restante da equipe.

Diferentes ideias são apresentadas em relação à escolarização desse grupo e uma delas trata de que a qualificação tende a afastar o ACS da sua principal função: ser um trabalhador que, por manter uma forte ligação com o território onde vive e trabalha, tem assim uma maior capacidade de transmitir conhecimentos e compreender os anseios da comunidade ${ }^{14}$.

Quando questionados sobre o tempo de atuação como profissional ACS no território, um deles está atuando entre 2-3 anos, outro entre 4-5 anos e quatro já atuam no território há mais de 5 anos.

0 fato de permanecer bastante tempo na função contribui muito para a continuidade do serviço dentro da equipe e comunidade, além de favorecer 
um maior entendimento sobre a sua função e área de atuação, fazendo com que os profissionais possam, ainda, construir estratégias baseadas nas necessidades dos usuários sob sua responsabilidade sanitária ${ }^{10}$.

A Tabela 2 apresenta as variáveis investigadas no questionário de identificação e prevenção dos riscos ocupacionais aos quais os ACS estão expostos em seu ambiente de trabalho.

Tabela 2. Exposição dos ACS aos riscos ocupacionais em seu ambiente de trabalho.

\begin{tabular}{|c|c|c|}
\hline Variável & $\mathbf{N}$ & $(\%)$ \\
\hline \multicolumn{3}{|c|}{ Você acha que está exposto a riscos durante seu trabalho? } \\
\hline Sim & 6 & 100 \\
\hline Não & 0 & 0 \\
\hline \multicolumn{3}{|c|}{ Você identifica riscos de acidente de trânsito durante a realização do seu trabalho? } \\
\hline Sim & 5 & 84 \\
\hline Não & 1 & 16 \\
\hline \multicolumn{3}{|c|}{ Você identifica riscos de adoecimento através do contato com o usuário? } \\
\hline Sim & 6 & 100 \\
\hline Não & 0 & 0 \\
\hline \multicolumn{3}{|c|}{ Você usa EPI } \\
\hline Sim & 0 & 0 \\
\hline Não & 6 & 100 \\
\hline \multicolumn{3}{|c|}{ Você usa protetor solar durante o trabalho? } \\
\hline Sim & 5 & 84 \\
\hline Não & 1 & 16 \\
\hline \multicolumn{3}{|c|}{ Você já sofreu algum acidente de trabalho? } \\
\hline Sim & 1 & 16 \\
\hline Não & 5 & 84 \\
\hline \multicolumn{3}{|c|}{ Afastamento do trabalho por problema de saúde relacionado ao mesmo? } \\
\hline Sim & 1 & 16 \\
\hline Não & 5 & 84 \\
\hline \multicolumn{3}{|c|}{ Identifica riscos de violência urbana para com você em seu território? } \\
\hline Sim & 6 & 100 \\
\hline Não & 0 & 0 \\
\hline \multicolumn{3}{|c|}{ Você se sente pressionado na realização do seu trabalho? } \\
\hline Sim & 2 & 33 \\
\hline Não & 4 & 67 \\
\hline \multicolumn{3}{|c|}{ Sente algum incômodo/dor durante o seu trabalho? } \\
\hline Sim & 4 & 67 \\
\hline Não & 2 & 33 \\
\hline \multicolumn{3}{|c|}{ Como você classifica seu conhecimento acerca da prevenção dos riscos relacionados ao trabalho? } \\
\hline Muito bom & 1 & 16 \\
\hline Bom & 4 & 67 \\
\hline Regular & 1 & 16 \\
\hline Ruim & 0 & 0 \\
\hline \multicolumn{3}{|c|}{ Como você classifica seus cuidados e medidas de prevenção dos riscos relacionados ao seu trabalho? } \\
\hline Muito bom & 1 & 16 \\
\hline Bom & 3 & 51 \\
\hline Regular & 2 & 33 \\
\hline Ruim & 0 & 0 \\
\hline
\end{tabular}

Fonte: Dados da pesquisa. 
Quanto à exposição a riscos durante a realização do seu trabalho, todos os ACS afirmaram que se sentem expostos. Quanto aos riscos de acidente de trânsito durante a realização do trabalho, cinco responderam que sim.

Todos os ACS responderam que identificam riscos de adoecimento em contato com o usuário durante as visitas. No entanto, todos eles afirmam não fazer uso de equipamentos de proteção individual.

Sobre o uso de protetor solar durante a realização do trabalho, cinco responderam que usam. Os ACS unanimemente identificaram riscos de violência urbana contra eles, dentro do território de atuação.

Quatro deles responderam que não se sentem pressionados para a realização do trabalho.

Quando perguntados sobre incômodos musculoesqueléticos durante o trabalho, apenas dois afirmaram não sentir nenhum tipo de incômodo, os outros quatro afirmaram sentir dor em pelo menos um ponto, tendo sido referidos os seguintes: dor nos ombros, cotovelos, joelhos, tornozelos, coluna torácica e lombar.

Quando questionados se já sofreram algum acidente de trabalho, apenas um respondeu que sim. A mesma quantidade de ACS - quatro - precisou se afastar do trabalho por conta de algum problema de saúde laboral.

0s ACS foram perguntados sobre como classificam seu conhecimento acerca da prevenção dos riscos relacionados ao seu trabalho: um classifica como muito bom, quatro como bom e um como regular. E, por fim, foram indagados sobre como classificam seus cuidados e medidas de prevenção dos riscos relacionados a trabalho. Um classificou como muito bom, três como bom e dois como ruim.

$\mathrm{Na}$ realização das entrevistas, todos os ACS participantes do presente estudo referiram estar expostos a riscos durante a realização do seu trabalho, tanto de acidentes de trânsito como de violência urbana, além de se considerarem suscetíveis ao adoecimento em contato direto com os usuários assistidos. Como aponta o ACS 1:

Assim, não é nem que eu veja como muitos riscos, mas eu acho que sim, sabe? [...] mesmo a gente que anda a pé acaba também ficando exposta a acidente, não é só quem vai de moto pra área (ACS 1).

De acordo com a Norma Regulamentadora 9 (NR $9)$, são considerados riscos ambientais os agentes físicos, químicos e biológicos existentes nos ambientes de trabalho que são capazes de causar danos à saúde do trabalhador ${ }^{15}$.

No entanto, apesar de reconhecerem a existência desses riscos, $100 \%$ dos participantes referem que não usam EPI e $16 \%$ referem não fazer uso de proteção solar, o que torna necessária a conscientização para que estes passem a executar essas medidas preventivas, tendo em vista que passam a maior parte do tempo expostos ao sol e em contato com outros agentes de risco.

A gente sabe que precisa usar, que é bom pra nós mesmos, mas aí acaba nem usando porque nunca deram pra gente (ACS 2).

A busca de uma justificativa para o não uso dos EPI pode estar no fato de que a gestão não oferece esse suporte aos ACS ${ }^{16}$. Segundo a NR 21, nos trabalhos realizados a céu aberto, devem ser exigidas medidas preventivas especiais que protejam os trabalhadores de frio, ventos inconvenientes, calor e insolação excessiva. 0 ACS realiza suas atividades laborais durante o tempo de maior incidência dos raios solares, o que torna essencial o uso do protetor solar, principalmente quando se trabalha em regiões mais quentes, como é o caso do cenário do estudo ${ }^{17}$. Todavia, não há uma priorização por parte dos próprios trabalhadores em relação a essa pauta e/ou reivindicação junto à gestão.

Assim como outros autores nos revelam, encontramos no presente estudo que o ACS possui entendimento e consciência sobre a importância do uso dos EPI, porém, na maioria das vezes, acaba não usando por esquecimento ${ }^{18}$. Fica, entretanto, o questionamento: o não uso se dá, de fato, por esquecimento ou esse agente de promoção acaba não considerando que existem riscos potenciais no desenvolvimento de suas ações laborais? É provável que esse agente de promoção à saúde não esteja dando importância efetiva às medidas preventivas.

0 território de atuação dos ACS entrevistados é de grande vulnerabilidade social, o que pode justificar o fato de todos os participantes da pesquisa relatarem que identificam riscos de violência urbana durante a realização do seu trabalho, tendo em vista que eles cotidianamente acessam áreas que contam com taxas consideráveis de miséria, homicídios e tráfico de drogas.

Com toda certeza existe risco. A gente sabe que nossa área é muito vulnerável e 
também tem a questão das facções aqui na área, né? (ACS 5).

0 reconhecimento desses aspectos da violência urbana acaba gerando uma postura de medo:

Olhe que a gente entra em todos os cantos porque é o nosso trabalho. Mas sempre tem o medo, né, justamente por conhecer todas as pessoas que moram aqui, a gente sabe onde pode ter mais perigo (ACS 3 ).

No Ceará, a violência tem crescido consideravelmente, o que contribui para que a visita domiciliar realizada pelo ACS represente um risco de vida ao profissional. Na cidade de Fortaleza, por exemplo, as consequências da violência urbana têm sido responsáveis por pedidos de afastamento de trabalhadores da saúde em locais de vulnerabilidade social ${ }^{19}$.

Ainda assim, atualmente, a ESF é uma estratégia bem preparada para enfrentar a violência urbana, isso devido ao seu processo dinâmico dentro dos territórios, sendo ainda capaz de desenvolver estratégias de acordo com uma visão ampliada de saúde daquele local. A violência urbana é um tema crescente no Brasil, no entanto ficando mais ligado ao campo da Segurança Pública e da Justiça, e ainda incipientemente debatido na saúde, apesar de influenciar diretamente a situação de saúde da população e os processos de trabalho ${ }^{20}$.

Alguns autores nos revelam ainda que além do risco de violência urbana, pode existir também a violência por parte dos usuários. A insatisfação dos usuários com o serviço de saúde e a falta de resolução de suas demandas contribuem para que eles respondam ao ACS, que é o profissional de contato direto e mais próximo, com agressões verbais e/ou pressão psicológica ${ }^{21}$.

No presente estudo, apenas dois dos participantes referem se sentir pressionados na realização do trabalho, concordando com o que é encontrado na literatura referente aos danos emocionais gerados pelo trabalho do $\mathrm{ACS}^{22}$.

Eu me sinto às vezes, principalmente com essa questão de recadastrar as famílias e o prazo de entrega dessas coisas muito em cima da hora que eles pedem pra gente (ACS $6)$.
Parece haver uma dupla carga de pressão: 0 serviço/a gestão e a população adscrita:

Quem mais pressiona a gente é o povo, sabia? Eles querem que a gente dê conta de coisa que não é da nossa capacidade, aí complica muito, viu? (ACS 4).

Quando essa demanda da população se encontra com a organização do processo de trabalho de uma equipe desarticulada e pouco resolutiva, essa percepção de pressão aumenta. Essa, porém, não é uma realidade unânime no cenário nacional, há muitas variações a partir do perfil de cada equipe e de cada UBS.

É inegável a importância e responsabilidade que o ACS tem dentro da equipe da ESF. São muitas as suas atribuições, configurando um processo de trabalho bastante singular, que está diretamente ligado às ações de promoção da saúde. No entanto, o trabalho real fica um pouco além do que thes é atribuído, tendo em vista que as demandas da comunidade se encontram quase sempre acima dos limites impostos no trabalho, fazendo com que o trabalhador ACS transcenda ao que se é preconizado como tarefas e carga horária de sua função $0^{22}$.

As lesões musculoesqueléticas relacionadas ao trabalho são tidas hoje como um problema de saúde mundial. Quanto ao acometimento musculoesquelético, quatro dos seis entrevistados relatam sentir incômodo em pelo menos um ponto no corpo. Esse achado corrobora outros estudos que nos revelam que os problemas de coluna estão entre os mais diagnosticados nos ACS 9 .

Além do acometimento musculoesquelético, os acidentes de trabalho também são tidos como um problema de saúde pública em âmbitos nacional e mundial, principalmente pelos danos causados à saúde e os altos índices de afastamento do trabalho ${ }^{23}$. No entanto, ainda existe uma grande escassez nos estudos referentes a esse assunto quando falamos de trabalhadores da Atenção Básica, onde o ACS está inserido. Dentre os ACS entrevistados, apenas um refere já ter sofrido acidente de trabalho e necessitado se afastar do trabalho por conta disso.

Segundo Almeida et al. ${ }^{23}$, em um estudo realizado em Sidrolândia-MS, a maioria dos acidentes que acometem os ACS são relacionados ao trânsito, devido à necessidade de se locomover para realizar suas atividades ou até mesmo na locomoção de casa para o trabalho. 
A maioria dos ACS entrevistados classificam seu conhecimento acerca dos riscos e as medidas de prevenção adotadas como bom. Observa-se a necessidade de mais estudos sobre esse tema, no entanto podemos versar no que diz respeito à capacitação dos ACS sobre a saúde do trabalhador e o processo saúde-doença. Pelo fato de ser um trabalho complexo, esses profissionais necessitam de um processo de educação permanente constante; percebe-se, porém, que vem se instituindo apenas processos de qualificação rápidos, que são demandados por questões pontuais em saúde ${ }^{24}$.

\section{CONCLUSÃO}

Este estudo possibilitou conhecer os riscos à saúde implicados pelo trabalho, bem como o conhecimento e a execução das medidas de prevenção a esses riscos por parte dos ACS de uma UBS do interior do Ceará.

Sabe-se da limitação deste estudo, uma vez que analisou uma pequena amostra dos ACS. Além disso, sabe-se que, ao responder ao questionário, os participantes podem ter entendimentos diferentes e limitados sobre o que é um risco ocupacional ou acidente de trabalho. Entretanto, acredita-se que esta pode ser uma importante contribuição ao publicizar a realidade de um município do interior do Ceará, fomentando o debate sobre as particularidades da organização da APS em diferentes contextos.

Evidenciou-se que o processo de trabalho desses ACS vem acarretando consequências em sua saúde e qualidade de vida, sendo muitos os riscos aos quais estão expostos esses trabalhadores da saúde. os principais riscos estão ligados aos aspectos ergonômicos e psicossociais existentes no ambiente laboral, no entanto a maioria afirma conhecer e adotar medidas de prevenção em relação aos riscos no trabalho. Algumas medidas básicas, como o uso de EPI e proteção solar, todavia, não fazem parte da rotina dos ACS. Isso nos leva a questionar sobre a concepção e priorização da prevenção na perspectiva desses indivíduos. Esse é um objeto de pesquisa que pode e deve ainda ser aprofundado em outras oportunidades.

Destaca-se a importância da realização de outras pesquisas semelhantes a fim de reforçar o conhecimento e respaldar medidas de segurança a essa classe trabalhadora, visto que esta pesquisa foi realizada com ACS pertencentes a uma única UBS.

\section{CONTRIBUIÇÃO DOS AUTORES}

Barbara Luma Araújo Nógimo e Gisele Maria Melo Soares Arruda contribuíram com a concepção da pesquisa, delineamento do estudo, análise e interpretação dos dados, bem como a redação deste manuscrito. Danielly Maia de Queiroz e Pedro Alves de Araújo Filho contribuíram com a revisão crítica e aprovação da versão a ser publicada.

\section{REFERÊNCIAS}

1. Arantes LJ, Shimizu HE, Merchán-Hamann E. Contribuições e desafios da Estratégia Saúde da Família na Atenção Primária à Saúde no Brasil: revisão da literatura. Ciênc Saúde Colet. 2016; 21(5):1499-1510.

2. Macinko J, Mendonça CS. Estratégia Saúde da Família, um forte modelo de Atenção Primária à Saúde que traz resultados. Saúde debate. 2018; 42(1):18-37.

3. Pinto LF, Giovanella L. Do Programa à Estratégia Saúde da Família: expansão do acesso e redução das internações por condições sensíveis à atenção básica (ICSAB). Ciênc Saúde Colet. 2018; 23(6):1903-1914.

4. Ávila MMM. 0 Programa de Agentes Comunitários de Saúde no Ceará: o caso de Uruburetama. Ciênc Saúde Colet. 2011; 16(1):349-360.

5. Alonso CMC, Béguin PD, Duarte FJCM. Work of community health agents in the Family Health Strategy: meta-synthesis. Rev Saúde Púb. 2018; $52: 14$.

6. Samudio JLP, Brant LC, Martins ACFDC, Vieira MA, Sampaio CA. Agentes comunitários de saúde na Atenção Primária no Brasil: multiplicidade de atividades e fragilização da formação. Trabalho, Educação e Saúde. 2017; 15(3):745-769.

7. Atlas do Censo Demográfico 2010. Referências. IBGE. MALHA municipal digital do Brasil: situação em 2000 e 2010. Rio de Janeiro: IBGE; 2012.

8. Brasil. Ministério da Saúde. Diretrizes e normas regulamentadoras sobre pesquisa envolvendo seres humanos. Resolução n 466, de 12 dez 2012. Brasília (DF); 2012.

9. Simas PRP, Pinto ICM. Trabalho em saúde: retrato dos agentes comunitários de saúde da região Nordeste do Brasil. Ciênc Saúde Colet.2017; 22(6):1865-1876.

10. Guimarães MSA, Sousa MF,Mucari TB. Perfil Sociodemográfico dos Agentes Comunitários de Saúde da Estratégia Saúde da Família no Município de Palmas-T0. Rev Desafios. 2017; 4(3):60-72. 
11. Pedraza DF, Santos I. Perfil e atuação do agente comunitário de saúde no contexto da Estratégia Saúde da Família em dois municípios da Paraíba. Interações (Campo Grande). 2017; 18(3):97105.

12. Garcia ACP, Lima RCD, Lima EFA, Galavote HS, Andrade MAC. Perfil y el Proceso de Trabajo de los Agentes Comunitarios de Salud. Rev Pesqui (Univ. Fed. Estado Rio J., Online). 2019; 11(2): 339-44.

13. Bandeira FC, Gonçalves LG. A dimensão educativa do trabalho do agente comunitário de saúde: um percurso pelas diretrizes. Comunicações Piracicaba. $2019 ; 26(3): 3-22$.

14. Cabral JF, Gleriano JS, Nascimento JDM. Perfil sociodemográfico e formação profissional de agentes comunitários de saúde. Rev Interdisciplinar de Estudos em saúde da UNIARP. 2019; 9(2):193-209.

15. Brasil. Norma Regulamentadora - 9. NR - 9 Programa de Prevenção de Riscos Ambientais. Brasília (DF): Ministério do Trabalho e Emprego; 2009.

16. Cândido AS, Ferreira RJ. Riscos à Saúde e à Segurança no Trabalho do Agente de Combate as Endemias do Município de Campos Sales, Ceará, Brasil. Ensaios Cienc, Cienc Biol Agrar Saúde. 2017; 21(1):52-57.

17. Junior ARF, Torres ARA, Silva CMA. Condições laborais dos agentes de combate a endemias e seus efeitos à saúde. Essentia. 2015; 16(esp):75-95.

18. Costa CMC, Costa LM, Silva BG, Moreira RS. Promovendo a saúde do trabalhador: enfoque no agente comunitário de saúde. In: Anais Congresso Brasileiro de Medicina de Família Comunidade; 2013; Belém, Brasil. Belém: CBMFC; 2013. p. 12:1554.

19. Barreto ICHC, Pessoa VM, Sousa MFA, Nuto SAS, Freitas RWJF, et al. Complexidade e potencialidade do trabalho dos Agentes Comunitários de Saúde no Brasil contemporâneo. Saúde debate. 2018; 42(esp 1):114-129.

20. Benício LFS, Barros JPP. Estratégia Saúde da Família e Violência Urbana: abordagens e práticas sociais em questão. Sanare (Sobral, Online). 2017; $16(1): 102-112$

21. Alonso CMC, Béguin PD, Duarte FJCM. Trabalho dos agentes comunitários de saúde na Estratégia Saúde da Família: metassíntese. Rev Saúde Pública. 2018; $52(14)$.

22. Santos AC, Hoppe AS, Krug SBF. Agente Comunitário de Saúde: implicações dos custos humanos laborais na saúde do trabalhador. Physis. $2019 ; 28(4):$ e280403.
23. Almeida MCS, Baptista PCP, Silva A. Acidentes de trabalho com agentes comunitários de saúde. Rev Enferm UERJ. 2016; 24(5).

24. Morosini MV, Fonseca AF. Os agentes comunitários na Atenção Primária à Saúde no Brasil: inventário de conquistas e desafios. Saúde debate. 2018; 42 (esp):261-274.
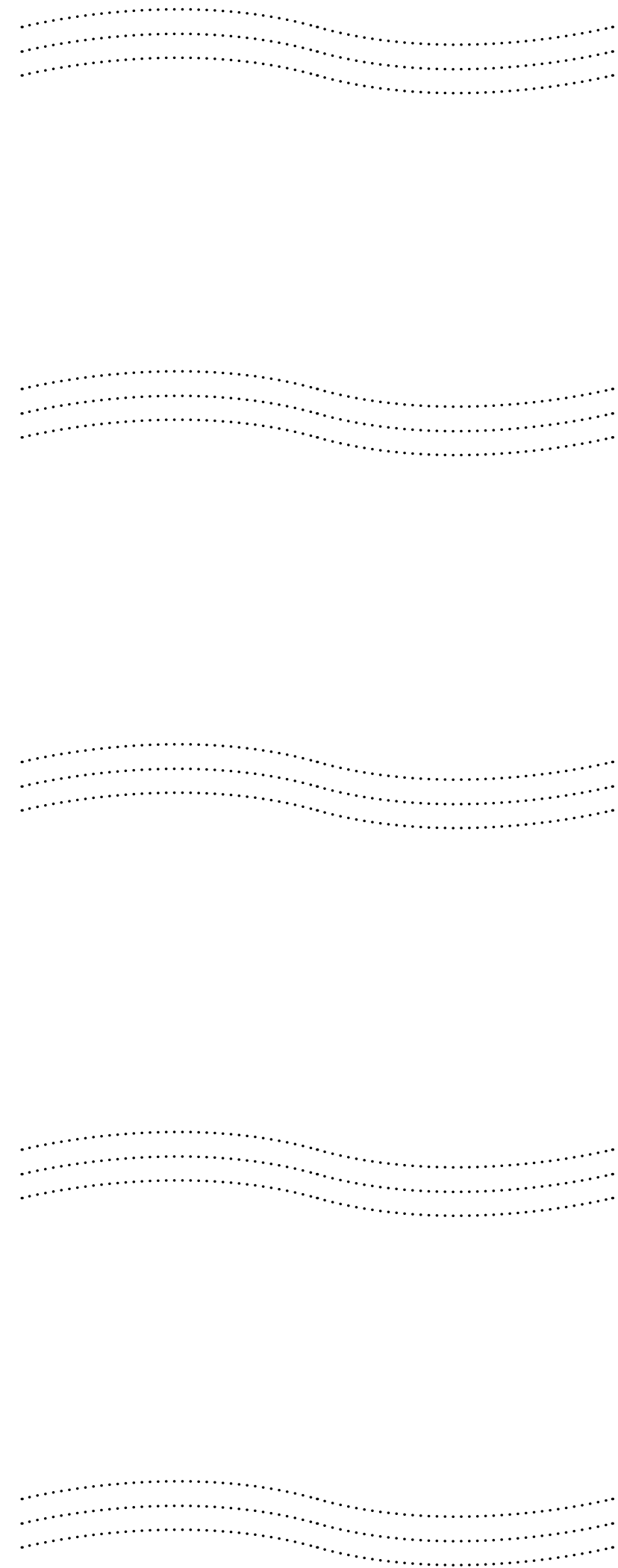